\title{
Author Correction: ALK phosphorylates SMAD4 on tyrosine to disable TGF- $\beta$ tumour suppressor functions
}

\begin{abstract}
Qianting Zhang (D), Mu Xiao, Shuchen Gu, Yongxian Xu, Ting Liu, Hao Li, Yi Yu, Lan Qin, Yezhang Zhu, Fenfang Chen, Yulong Wang, Chen Ding, Hongxing Wu, Hongbin Ji, Zhe Chen, Youli Zu, Stephen Malkoski, Yi Li®D, Tingbo Liang, Junfang Ji, Jun Qin, Pinglong Xu@ Bin Zhao, Li Shen, Xia Lin and Xin-Hua Feng (D)
\end{abstract}

Correction to: Nature Cell Biology https://doi.org/10.1038/s41556-018-0264-3, published online 21 January 2019

In the version of this Article originally published, several labels in Figs. 1b,e and 2c were incorrect. In the top HA-SMADs row of Fig. 1b, the ' 1 ' should be a minus sign. In Fig. 1e, the top plus sign in the 'in vitro' column should read 'n.d.', and the bottom 'n.d.' label in the 'co-IP' column should be a minus sign. In fig. 2c, the middle plus sign in the top 'His-SMAD4' row should be a minus sign. Additionally, grant number ' 31730057 ' was omitted from the Acknowledgements section. The last sentence of the section should read 'This research was partly supported by grants from NSFC (31730057, 91540205, 31571447, 31090360), NIH (R21CA209007) and DoD (DAMD W81XWH-15-1-0650/0651), and the Fundamental Research Funds for the Central Universities.” The errors have been corrected.

Published online: 2 February 2021

https://doi.org/10.1038/s41556-021-00638-5

(๑) Springer Nature Limited 2021

\section{Author Correction: Optogenetic manipulation of cellular communication using engineered myosin motors}

Zijian Zhang, Nicolas Denans (D), Yingfei Liu, Olena Zhulyn, Hannah D. Rosenblatt, Marius Wernig (D) and Maria Barna (D) Correction to: Nature Cell Biology https://doi.org/10.1038/s41556-020-00625-2, published online 1 February 2021

In the version of this Technical Report originally published, there was an error in the right panel of Fig. 2a. The arrow in the far-right image of optoATV - should be going from "+" to "-", pointing the image to the left instead of from "-" to "+". The error has been corrected.

Published online: 19 February 2021

https://doi.org/10.1038/s41556-021-00650-9

() The Author(s), under exclusive licence to Springer Nature Limited 2021

\section{Publisher Correction: The T-box transcription factor Eomesodermin governs haemogenic competence of yolk sac mesodermal progenitors}

Luke T. G. Harland, Claire S. Simon (D), Anna D. Senft D, Ita Costello D, Lucas Greder, Ivan Imaz-Rosshandler, Berthold Göttgens D, John C. Marioni (D), Elizabeth K. Bikoff, Catherine Porcher (D), Marella F. T. R. de Bruijn (D) and Elizabeth J. Robertson (D)

Correction to: Nature Cell Biology https://doi.org/10.1038/s41556-020-00611-8, published online 8 January 2021

In the version of this Article originally published, two colors in the key for Fig. 7a were incorrect. The circles for 'allantois' and 'endothelium' should be purple and green, respectively, instead of shades of grey. The errors have been corrected.

Published online: 11 February 2021

https://doi.org/10.1038/s41556-021-00645-6

(c) The Author(s), under exclusive licence to Springer Nature Limited 2021 